\title{
Energia alternativa de biomassa: Bioetanol a partir da casca e da polpa de banana
}

\author{
Ozair Souza ${ }^{1,2}$, Marco A. Schulz ${ }^{2}$, Gustavo A. A. Fischer ${ }^{1}$, \\ Theodoro M. Wagner ${ }^{1}$ \& Noeli Sellin ${ }^{1,2}$
}

\begin{abstract}
RESUMO
A conversão de biomassas agroindustriais em bioetanol com consequente valorização de rejeitos e resíduos, tem sido objeto de estudos de várias pesquisas realizadas no Brasil e no mundo. Neste trabalho foi avaliada a potencialidade do uso da polpa e da casca da banana (Musa cavendishii), tanto in natura como previamente hidrolisada por ácido e enzimas, como substrato da fermentação alcoólica. Os rendimentos médios em bioetanol (em base úmida de biomassa) obtidos com a polpa $(0,48 \pm 0,05$ $\left.\mathrm{g} \mathrm{g}^{-1}\right)$ e com a casca $\left(0,34 \pm 0,11 \mathrm{~g} \mathrm{~g}^{-1}\right)$, ambos in natura, possibilitaram a eficiência do processo de conversão, da ordem de $95 \%$ do rendimento teórico. A produtividade máxima alcançada em bioetanol foi de 3,0 $\pm 0,7 \mathrm{~g} \mathrm{~L}^{-1} \mathrm{~h}^{-1}$ com o uso da polpa e de 1,32 $\pm 0,03 \mathrm{~g} \mathrm{~L}^{-1} \mathrm{~h}^{-1}$ com a casca. Nas condições operacionais avaliadas o pretratamento dos resíduos com ácido sulfúrico não é recomendado para a produção de bioetanol.
\end{abstract}

Palavras-chave: bioálcool, biocombustíveis, resíduos agrícolas

\section{Alternative energy from biomass: Bioethanol from banana pulp and peels}

\begin{abstract}
The conversion of agroindustrial biomasses in bioethanol with consequent enrichment of wastes has been the object of various research projects conducted in Brazil and around the world. This study evaluated the potential of the Musa cavendishii banana pulp and peels using in natural state and also waste previously hydrolyzed by acid and enzimes, as substrate of alcoholic fermentation. The mean bioethanol yields (on wet biomass base), obtained with the pulp $\left(0.48 \pm 0.05 \mathrm{~g} \mathrm{~g}^{-1}\right)$ and with the peels $\left(0.34 \pm 0.11 \mathrm{~g} \mathrm{~g}^{-1}\right)$, both in natural state, enabled a conversion process efficiency to the order of $95 \%$ of theoretical yield. Maximum value reached in bioethanol was $3.0 \pm 0.7 \mathrm{~g} \mathrm{~L}^{-1} \mathrm{~h}^{-1}$ with pulp and $1.32 \pm$ $0.03 \mathrm{~g} \mathrm{~L}^{-1} \mathrm{~h}^{-1}$ with peels. Under the evaluated operating conditions, the pre-treatment of wastes with sulfuric acid is not recommended for bioethanol production.
\end{abstract}

Key words: bioalcohol, biofuels, agricultural waste 


\section{INTRODUÇÃO}

Devido os compromissos mais sólidos assumidos com a questão ambiental desde a assinatura do Protocolo de Quioto e em função de ser o petróleo, principal fonte de energia utilizada hoje, um combustível fóssil e não renovável, uma das maiores preocupações do mundo atual tem sido o suprimento de energia nas próximas décadas, razão por que fizeram renascer a atenção quanto às fontes alternativas de energia.

Existem diversas fontes alternativas de energia que, embora não possam substituir o petróleo em sua totalidade, podem contribuir para diminuir o consumo. A geração de energia a partir de biomassa tem sido objeto de vários estudos (Yu et al., 2002; Leite et al., 2003; Gonçalves et al., 2009; Souza et al., 2010; Quadros et al., 2010).

Qualquer matéria-prima orgânica passível de ser transformada em energia pode ser classificada como biomassa e, de acordo com sua origem, pode ser florestal (madeira), agrícola (soja, arroz e cana-de-açúcar, entre outras) ou oriunda de rejeitos urbanos ou industriais, sólidos ou líquidos (ANEEL, 2008).

Os rejeitos e os resíduos agrícolas, em sua maioria contendo materiais lignocelulósicos, ocupam lugar de destaque entre a biomassa disponível sobretudo em função da sua abundância e do caráter renovável. A biomassa lignocelulósica é composta, basicamente, por 40 a $60 \%$ de celulose, 20 a $40 \%$ de hemicelulose e 15 a 25\% de lignina (Moreira, 2005).

A disponibilidade de palhas, folhas, resíduo de exploração madeireira, rejeitos agrícolas e outros, tem despertado o interesse para seu uso como matéria-prima na produção de bioetanol, mais precisamente o etanol de segunda geração (Mojovic et al., 2006; Sassner et al., 2006; Sharma et al., 2007), processo no qual os açúcares fermentescíveis, por não se encontrarem solúveis como na fermentação do caldo de canade-açúcar (etanol de primeira geração), precisam ser previamente hidrolisados de seus polímeros celulose e hemicelulose. No entanto, a necessidade da obtenção de altas concentrações de açúcares fermentecíveis livres sem, contudo, gerar elementos tóxicos à fermentação, tem dificultado o emprego desses resíduos (Cardona \& Sánchez, 2007; Solomon et al., 2007). Segundo Balat (2011) as rotas mais estudadas e indicadas para a despolimerização de substratos lignocelulósicos têm sido a hidrólise ácida e a hidrólise enzimática, com pré-tratamento físico (explosão a vapor) ou químico (solução de $\mathrm{NaOH}$ ).

O Brasil se destaca como um dos maiores produtores agrícolas do mundo e, em consequência disto, é capaz de gerar grandes quantidades de rejeitos e resíduos agroindustriais. Entre essa biomassa se encontra a da bananicultura, a qual, segundo Souza et al. (2010), além dos frutos rejeitados para comercialização, para cada tonelada de banana colhida aproximadamente três toneladas de resíduos são gerados, entre os quais a casca do fruto industrializado, o pseudocaule, as folhas e o engaço da bananeira.

Todos esses resíduos estão sendo avaliados pela Universidade da Região de Joinville - Univille na geração de energia e, como parte integrante desse estudo, este trabalho trata especificamente do uso da polpa e casca da banana como substrato da fermentação alcoólica. Foram determinadas as propriedades físico-químicas dos resíduos in natura, avaliada a influência de diferentes métodos de hidrólise sobre a despolimerização da celulose e, a partir das condições operacionais ideais de pretratamento, estimados os valores de rendimento, produtividade e eficiência da fermentação.

A produção de bioetanol a partir dessas biomassas tornase um processo bastante atraente pois, além de permitir a criação de uma fonte alternativa e renovável de energia e de contribuir com a redução de resíduos no meio ambiente, a geração de energia pode agregar valor à matriz produtiva da fruta reduzindo, assim, o risco de perdas provocado por sua comercialização.

\section{Material e MÉTODOS}

As biomassas utilizadas foram a polpa e a casca da banana Musa cavendishii, popularmente conhecida no sul do Brasil como banana nanica. Os resíduos foram coletados numa indústria da região, cortados em pedaços de 1-2 cm e homogeneizados em liquidificador doméstico de $2 \mathrm{~L}$, em meio aquoso, na concentração de $250 \mathrm{~g} \mathrm{~L}^{-1}$ de massa úmida de biomassa ( $\mathrm{g} \mathrm{MU} \mathrm{L}^{-1}$ ). Esta concentração é correspondente a $74,8 \mathrm{~g} \mathrm{~L}^{-1}$ de massa seca $\left(\mathrm{g} \mathrm{MS} \mathrm{L}^{-1}\right.$ ) para a polpa e $28,5 \mathrm{~g} \mathrm{MS} \mathrm{L}^{-1}$ para a casca.

Foram realizadas duas séries de ensaios cada uma contendo dezoito ensaios de pré-tratamento para cada um dos resíduos avaliados. Em ambos os casos foi utilizado, como testemunha, o resíduo in natura, na temperatura ambiente e sem adição de ácido. Como reatores foram utilizados frascos Erlenmeyer de $250 \mathrm{~mL}$ contendo $100 \mathrm{~mL}$ de volume de trabalho $\left(\mathrm{V}_{\mathrm{T}}\right)$ e biomassa na concentração de $250 \mathrm{~g} \mathrm{MU} \mathrm{L}^{-1}$. A Tabela 1 apresenta as diferentes condições operacionais avaliadas.

Os ensaios a 100 e $120^{\circ} \mathrm{C}$ foram conduzidos em autoclave a vapor e a $90{ }^{\circ} \mathrm{C}$, em banho termostatizado.

Tabela 1. Ensaios de hidrólise ácida (HA) da polpa e da casca da banana nanica

\begin{tabular}{|c|c|c|c|c|c|c|c|c|c|}
\hline \multirow{2}{*}{ Condições experimentais } & \multicolumn{9}{|c|}{ Ensaios* } \\
\hline & $H A_{0,90,15}$ & $H A_{0,90,30}$ & $\mathrm{HA}_{1,90,15}$ & $\mathrm{HA}_{1,90,30}$ & $\mathrm{HA}_{2,90,15}$ & $\mathrm{HA}_{2,90,30}$ & $\mathrm{HA}_{0,100,15}$ & $\mathrm{HA}_{0,100,30}$ & $\mathrm{HA}_{1,100,15}$ \\
\hline $\mathrm{H}_{2} \mathrm{SO}_{4}\left(\% \mathrm{~kg} \mathrm{~kg}^{-1}\right)$ & 0 & 0 & 1 & 1 & 2 & 2 & 0 & 0 & 1 \\
\hline Temperatura $\left({ }^{\circ} \mathrm{C}\right)$ & 90 & 90 & 90 & 90 & 90 & 90 & 100 & 100 & 100 \\
\hline \multirow[t]{2}{*}{ Tempo de reação (min) } & 15 & 30 & 15 & 30 & 15 & 30 & 15 & 30 & 15 \\
\hline & $\mathrm{HA}_{1,100,30}$ & $\mathrm{HA}_{2,100,15}$ & $\mathrm{HA}_{2,100,30}$ & $\mathrm{HA}_{0,120,15}$ & $\mathrm{HA}_{0,120,30}$ & $\mathrm{HA}_{1,120,15}$ & $\mathrm{HA}_{1,120,30}$ & $\mathrm{HA}_{2,120,15}$ & $\mathrm{HA}_{2,120,30}$ \\
\hline $\mathrm{H}_{2} \mathrm{SO}_{4}\left(\% \mathrm{~kg} \mathrm{~kg}^{-1}\right)$ & 1 & 2 & 2 & 0 & 0 & 1 & 1 & 2 & 2 \\
\hline Temperatura $\left({ }^{\circ} \mathrm{C}\right)$ & 100 & 100 & 100 & 120 & 120 & 120 & 120 & 120 & 120 \\
\hline Tempo de reação (min) & 30 & 15 & 30 & 15 & 30 & 15 & 30 & 15 & 30 \\
\hline
\end{tabular}

* Os números subscritos nas identificações dos ensaios representam, sequencialmente: concentração de ácido, temperatura do processo e tempo de reação. Como testemunha foi utilizado o resíduo in natura, na temperatura ambiente e sem adição de ácido 
A partir dos resultados da hidrólise os resíduos foram fermentados em biorreator de bancada (ensaios F) e, como ensaio padrão de fermentação (testemunha), foi utilizada glicose $\left(20 \mathrm{~g} \mathrm{~L}^{-1}\right)$ em substituição ao resíduo a ser avaliado. Todos os ensaios foram realizados com uma replicata sendo retiradas, a cada $2 \mathrm{~h}$, duas amostras para determinação das concentrações médias de açúcares totais (AT) e etanol (P).

Para obtenção do inóculo e realização do ensaio padrão de fermentação (ensaio FGY), foi utilizado o meio de cultivo, aqui denominado GY, o qual foi preparado a partir dos meios propostos por Pamarola-Adrados et al. (2005) e Saito \& Cabello (2006). As concentrações de nutrientes do meio GY foram de (em g L $\left.{ }^{-1}\right)$ : glicose, 20; extrato de levedura, 3; $\left(\mathrm{NH}_{4}\right)_{2} \mathrm{SO}_{4}, 0,5$; $\mathrm{K}_{2} \mathrm{HPO}_{4}, 0,5 ; \mathrm{MgSO}_{4} \cdot 7 \mathrm{H}_{2} \mathrm{O}, 0,1$ e $\mathrm{CaCl}_{2}, 0,1$.

Para os ensaios de fermentação empregando-se a polpa (ensaios FPol) ou a casca de banana (FCas) como substrato, o meio base de cultivo, previamente esterilizados em autoclave a $120^{\circ} \mathrm{C}$ durante $15 \mathrm{~min}$, foi o mesmo do inóculo, exceto a glicose, que foi substituída pelo resíduo avaliado.

Foi empregada a levedura Saccharomyces cerevisiae isolada de fermento comercial seco; o microorganismo foi mantido por meio de repiques semanais em cultivo de superfície, empregando-se placas de Petri com meio GY adicionado de 20 $\mathrm{g} \mathrm{L}^{-1}$ de Ágar-agar.

O inóculo foi produzido em frasco de $500 \mathrm{~mL}$ contendo 190 $\mathrm{mL}$ do meio de cultivo GY e $10 \mathrm{~mL}$ de suspensão microbiana obtida de quatro placas de Petri, do cultivo de manutenção; a mistura foi incubada em agitador orbital com frequência de agitação de $120 \mathrm{~min}^{-1}, 30{ }^{\circ} \mathrm{C}$, durante $18 \mathrm{~h}$.

Para os ensaios em fermentador de bancada (ensaios F) foi utilizado o biorreator Biostat ${ }^{\circledR} \mathrm{B}$ da B. Braun com dorna de $5 \mathrm{~L}$ e volume de trabalho de $2 \mathrm{~L}$ contendo $20 \% \mathrm{v} / \mathrm{v}$ de inóculo. Experimentos realizados: 1 ensaio padrão com glicose $20 \mathrm{~g} \mathrm{~L}^{-1}$ (Ensaio FGY); 3 ensaios com polpa de banana in natura, nas concentrações de 250, 375 e $500 \mathrm{~g} \mathrm{MU} \mathrm{L}^{-1}\left(\mathrm{FPol}_{250}, \mathrm{FPol}_{375} \mathrm{e}\right.$ $\mathrm{FPol}_{500}$ ) e 2 ensaios com casca de banana in natura nas concentrações de 250 e 1210 g MUL $^{-1}\left(\mathrm{FCas}_{250}\right.$ e FCas $\left.{ }_{1210}\right)$. Para o ensaio $\mathrm{FCas}_{1210}$, devido à alta concentração de sólidos, a casca foi previamente cozida em água fervente, na proporção de $1 \mathrm{~mL}$ de água para cada $2 \mathrm{~g}$ de massa úmida de resíduo, durante 30 min, seguida da filtração em malha de algodão para obtenção de caldo de fermentação. $\mathrm{O}$ acréscimo do gasto energético devido à operação de cozimento em comparação ao uso do resíduo não cozido, não foi objeto deste estudo e deverá ser avaliado em estudo posterior.

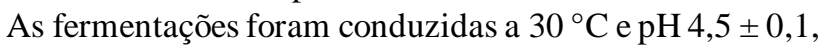
controlado automaticamente pela adição de $\mathrm{KOH} 1 \mathrm{M} \mathrm{e} \mathrm{HCl}$ 1M. Para a agitação foram empregados frequência de $150 \mathrm{~min}^{-1} \mathrm{e}$ sistema de agitação composto de duas turbinas espaçadas 20 $\mathrm{mm}$, contendo cada uma seis pás planas (flat-blade) de diâmetro $65 \mathrm{~mm}$, comprimento $18 \mathrm{~mm}$ e largura de $12 \mathrm{~mm}$. A distância da última turbina ao fundo da dorna foi de $45 \mathrm{~mm}$.

A determinação do teor de umidade foi realizada por gravimetria: em cadinhos limpos, previamente secos até massa constante, foram adicionados $3 \mathrm{~g}$ de amostra úmida (MU) e secadas a $105^{\circ} \mathrm{C}$ durante $24 \mathrm{~h}$; em seguida, as amostras foram resfriadas em dessecador e pesadas para determinação da massa seca (MS); com os valores encontrados utilizou-se a Eq. 1 para o cálculo da umidade.

$$
\text { Umidade }(\%)=\frac{M U-M S}{M U} \times 100
$$

Foram realizadas no Laboratório de Bromatologia da Faculdade de Zootecnia e Engenharia de Alimentos da Universidade de São Paulo, Pirassununga, SP. O teor de lignina foi determinado pelo método de Klason em permanganato de potássio e a celulose indiretamente, a partir dos valores do teor de fibra em detergente ácido (FDA) e teor de fibra em detergente neutro livre de cinzas (FDN), conforme descrito por Silva \& Queiroz (2002) e Soest (1967).

As amostras foram previamente centrifugadas a $4000 \mathrm{~min}^{-}$ ${ }^{1}$ durante 4 min em centrífuga Biofuge, contendo rotor 7591; após a centrifugação o sobrenadante foi filtrado em membrana de $45 \mu \mathrm{m}$ e analisado em cromatografia líquida de alta eficiência (HPLC); prosseguindo, foram empregados o detector de índice de refração Merck, modelo RI-71 e a coluna Eurokat $\mathrm{Pb}$. Os valores de açúcares totais (AT) foram obtidos a partir da soma dos carboidratos glicose (Glc), frutose (Frt) e sacarose ( $\mathrm{Scr})$.

De cada ensaio de fermentação foram retiradas amostras periódicas para as determinações das concentrações AT, conforme metodologia descrita anteriormente, e de etanol (P); para $\mathrm{P}$ foram empregadas a cromatografia gasosa (CG) utilizando-se cromatógrafo Agilent, 6890 e a coluna da Hewlett-Packard HP-1 com fase estacionária $100 \%$ dimetil polisiloxano.

Os valores de rendimento em açúcares totais obtidos nos ensaios de hidrólise ácida foram calculados de acordo com a Eq. 2.

$$
\mathrm{R}=\frac{\mathrm{AT}-\mathrm{AT}^{0}}{\mathrm{MU}} \times 100
$$

$\mathrm{R}$ - rendimento percentual em AT, \%

AT - concentração de açúcares totais no caldo após tratamento, $\mathrm{g} \mathrm{L}^{-1}$

$\mathrm{AT}^{0}$ - concentração de AT no caldo sem tratamento: ensaio $\mathrm{HA}_{0,0,0}, \mathrm{~g} \mathrm{~L}^{-1}$

MU- massa úmida de biomassa, $250 \mathrm{~g} \mathrm{~L}^{-1}$

Os valores de rendimento das fermentações representados pelo fator de conversão de açúcares totais em etanol $\left(\mathrm{Y}_{\mathrm{P} / \mathrm{AT}}\right)$ foram calculados de acordo com a Eq. 3.

$$
Y_{P / A T}=\frac{\left(P_{f}-P_{0}\right)}{\left(A T^{0}-A T_{f}\right)}
$$

\footnotetext{
$\mathrm{P}_{\mathrm{f}}$ - concentração de etanol no tempo final de fermentação, $\mathrm{g} \mathrm{L}^{-1}$

$\mathrm{P}_{0}$ - concentração de etanol no início do processo fermentativo, $\mathrm{g} \mathrm{L}^{-1}$

$\mathrm{AT}_{0}$ - concentração inicial de açúcares no início da fermentação, $\mathrm{g} \mathrm{L}^{-1}$
} 
$\mathrm{AT}_{\mathrm{f}}$ - concentração de açúcares no tempo $\mathrm{t}_{\mathrm{f}}, \mathrm{g} \mathrm{L}^{-1}$

$t_{f} \quad$ - tempo de fermentação correspondente à obtenção da máxima concentração de etanol no caldo fermentado, $h$

Os valores de produtividade total em etanol obtidos nas fermentações $\left(Q_{p}\right)$ foram expressos em massa do produto formado por unidade de tempo e por unidade de volume ( $\left.\mathrm{g} \mathrm{h}^{-1} \mathrm{~L}^{-1}\right)$ e obtidos pela Eq. 4, conforme definição de Gaden Jr. (1959).

$$
Q_{P}=\frac{P_{f}-P_{0}}{t_{f}}
$$

Os valores de rendimento em produto foram comparados aos do rendimento teórico máximo obtido nas fermentações alcoólicas (51,11\%), conforme Sharma et al. (2007), para se obter a eficiência do processo, de acordo com a Eq. 5.

$$
\varepsilon=\frac{\mathrm{Y}_{\mathrm{P} / \mathrm{AT}}}{0,5111} \times 100
$$

As duplicatas dos ensaios de fermentação foram analisadas pelo método ANOVA com teste de Tukey para $\mathrm{P}<0,05$ empregando-se o programa computacional Origin 7.5.

\section{RESULTADOS E DISCUSSÃO}

As propriedades físico-químicas da polpa e da casca da banana Musa cavendishii são apresentadas na Tabela 2.

Tabela 2. Propriedades físico-químicas da polpa e da casca da banana Musa cavendishii madura

\begin{tabular}{lcc}
\hline \multirow{2}{*}{ Parâmetro } & \multicolumn{2}{c}{ \% em base de massa úmida (\% MU) } \\
\cline { 2 - 3 } & Polpa & Casca \\
Umidade & $70,1 \pm 0,1$ & $88,6 \pm 0,2$ \\
Lignina & $2,2 \pm 0,1$ & $2,05 \pm 0,4$ \\
Celulose & $3,98 \pm 0,08$ & $2,18 \pm 0,03$ \\
Sacarose & $4,1 \pm 0,4$ & $0,1 \pm 0,1$ \\
Glicose & $7,8 \pm 1,4$ & $1,1 \pm 0,3$ \\
Frutose & $7,3 \pm 1,9$ & $1,1 \pm 0,3$ \\
\hline
\end{tabular}

Os valores de umidade apresentados na Tabela 2, tanto para a polpa quanto para as cascas, foram próximos daqueles encontrados no Atlas NAS (do inglês, Atlas of Nutritional Data on United States and Canadian Feeds), citados por Hammond et al. (1996): 75,7 e 83,8\%, respectivamente; as demais propriedades diferiram de outros valores encontrados na literatura. Sharma et al. (2007) caracterizaram as cascas de bananas residuais provenientes de uma indústria de alimentos da Índia e determinaram o teor de celulose de 28,67\% em base de massa seca (\% MS) e a concentração de açúcares redutores totais de $36,83 \mathrm{~g} \mathrm{~kg}^{-1} \mathrm{MS}$. Considerando o teor de umidade de $83,8 \%$ referenciado no Atlas NAS, esses valores seriam da ordem de 4,65 e $0,6 \%$ em base de massa úmida (\% MU). Arredondo et al. (2009) apresentaram valor de açúcar para a polpa, de apenas 4,3 $\pm 0,6 \% \mathrm{MS}$, ou seja, 1,1\% MU considerando o teor de umidade de $74,6 \%$, dado pelo autor. Acredita-se que, neste caso e em função do alto teor de amido encontrado pelo autor na amostra analisada $(53,2 \%)$, o mesmo tenha feito a caracterização da polpa verde e não da polpa madura, como apresentado na Tabela 2 além de que o autor não especificou o tipo de açúcar a que se referiu nem o método analítico empregado para tal. De acordo com Mohapatra et al. (2010), quanto mais madura a fruta maior o teor de açúcar na polpa e menor a presença de amido. Hammond et al. (1996) afirmam que na banana verde quase a totalidade do carboidrato não-estrutural da fruta está na forma de amido mas, durante seu amadurecimento, rapidamente é convertida em açúcar.

Com o objetivo de aumentar a quantidade de açúcares fermentescíveis disponíveis para a fermentação alcoólica, a polpa e a casca de banana foram previamente submetidas à sacarificação ácida. A Tabela 3 apresenta os valores de rendimento $(\mathrm{R})$ em AT obtidos após a hidrólise ácida dos resíduos com $\mathrm{H}_{2} \mathrm{SO}_{4}$.

Conforme se observar na Tabela 3 , o uso apenas do aquecimento a $120{ }^{\circ} \mathrm{C}$ durante 15 ou $30 \mathrm{~min}$, sem a adição de ácido (ensaios $\mathrm{HA}_{0,120,15}$ e $\mathrm{HA}_{0,120,30}$ ), proporcionou rendimento em base úmida, da ordem de $26 \%$ para a polpa e de $4 \%$ para as cascas. Com o emprego de ácido sulfúrico os valores máximos de rendimento $(34,10 \%$ para a polpa e $5,39 \%$ para as cascas $)$ foram alcançados com o uso de $\mathrm{H}_{2} \mathrm{SO}_{4} 2 \%, 120{ }^{\circ} \mathrm{C}, 15 \mathrm{~min}$ (ensaio $\mathrm{HA}_{2,120,15}$ ).

Com a hidrólise ácida da polpa e da casca de banana realizada foi possível obter, no caldo hidrolisado, no máximo 0,34 e $0,05 \mathrm{~g}$ AT para cada unidade de massa úmida tratada ( $\left.\mathrm{g} \mathrm{AT} \mathrm{g}^{-1} \mathrm{MU}\right)$, respectivamente, enquanto no caso do aquecimento desses resíduos sem ácido, referidos valores foram da ordem de 0,26 e $0,04 \mathrm{~g} \mathrm{AT} \mathrm{g}^{-1} \mathrm{MU}$. Este pequeno aumento proporcionado com a adição de $\mathrm{H}_{2} \mathrm{SO}_{4}$ no preparo do caldo precisa ser analisado com cuidado visto que análises cromatográficas de massa realizadas posteriormente revelaram a presença de 5-hidroximetilfurfural (5-HMF), nos caldos hidrolisados $\mathrm{com}_{2} \mathrm{SO}_{4} 2$ a $120{ }^{\circ} \mathrm{C}$, tanto no tempo de reação de $15 \mathrm{~min}$ quanto de $30 \mathrm{~min}$. Este composto, gerado a partir da hidrólise da celulose em condições mais severas, é inibidor da fermentação alcoólica sendo responsável pela ocorrência de uma cinética lenta com rendimento e produtividade limitados (Campo et al., 2006). Consequentemente, haveria a necessidade de submeter o caldo hidrolisado a um processo de purificação antes de ser conduzido à fermentação aumentando, assim, o custo final do bioetanol a ser produzido.

Recomenda-se, então, dentro das condições experimentais avaliadas, o uso das biomassas in natura apenas aquecidas (sem ácido para a fermentação).

O consumo de açúcares totais e a produção de bioetanol obtidos no ensaio padrão FGY e na fermentação da polpa 500 g MU L-1 (ensaio FPol $_{500}$ ) são mostrados nas Figuras 1A e 1B, respectivamente; comportamento cinético similar foi observado nos demais ensaios de fermentação realizados.

Conforme a Figura 1B, o tempo de fermentação (h) necessário para o consumo total da glicose no ensaio padrão FGY foi de apenas $6 \mathrm{~h}$ mas em trabalho semelhante Palmarola-Adrados et al. (2005) precisaram de $20 \mathrm{~h}$ de fermentação para o consumo total de glicose. Além de terem utilizado maior concentração inicial de glicose no meio de fermentação $\left(30 \mathrm{~g} \mathrm{~L}^{-1}\right)$ do que a 
Tabela 3. Concentração de açúcares totais (AT) obtidos no pretratamento da polpa madura (P) e da casca (C) de banana nanica e seus respectivos valores de rendimento $(\mathrm{R})$

\begin{tabular}{|c|c|c|c|c|c|}
\hline Ensaio* & $\begin{array}{l}\mathrm{AT}^{\star *} \\
\left(\mathrm{~g} \mathrm{~L}^{-1}\right)\end{array}$ & $\begin{array}{c}R^{\star *} \\
(\% \mathrm{MU})\end{array}$ & Ensaio* & $\begin{array}{l}A T^{\star *} \\
\left(g^{-1}\right)\end{array}$ & $\begin{array}{c}R^{* *} \\
(\% \mathrm{MU})\end{array}$ \\
\hline Resíduo in natura & $43,12 / 59,58$ & $17,25 / 23,83$ & $\mathrm{C}-\mathrm{HA} \mathrm{A}_{0,0,0}$ & $4,62 / 8,68$ & $1,85 / 3,47$ \\
\hline P-HA & 41,19 & 16,48 & $\mathrm{C}-\mathrm{HA}_{0,90,15}$ & 7,39 & 2,96 \\
\hline $\mathrm{P}-\mathrm{HA} \mathrm{A}_{0,90,30}$ & 47,45 & 18,98 & $\mathrm{C}-\mathrm{HA}_{0,90,30}$ & 6,13 & 2,45 \\
\hline P-HA $0,100,15$ & 53,81 & 21,52 & C-HA $\mathrm{H}_{0,100,15}$ & 6,88 & 2,75 \\
\hline $\mathrm{P}-\mathrm{HA} \mathrm{A}_{0,100,30}$ & 48,03 & 19,21 & $\mathrm{C}-\mathrm{HA}_{0,100,30}$ & 6,65 & 2,66 \\
\hline P-HA $A_{0,120,15}$ & $55,78 / 66,08$ & $22,31 / 26,43$ & $\mathrm{C}-\mathrm{HA}_{0,120,15}$ & $7,69 / 10,64$ & $3,08 / 4,26$ \\
\hline $\mathrm{P}-\mathrm{HA} \mathrm{A}_{0,120,30}$ & $54,21 / 65,90$ & $21,68 / 26,36$ & $\mathrm{C}-\mathrm{HA}_{0,120,30}$ & $7,70 / 10,26$ & $3,08 / 4,10$ \\
\hline $\mathrm{P}-\mathrm{HA}_{1,90,15}$ & 50,95 & 20,38 & $\mathrm{C}-\mathrm{HA}_{1,90,15}$ & 5,27 & 2,11 \\
\hline $\mathrm{P}-\mathrm{HA}_{1,90,30}$ & 61,81 & 24,72 & $\mathrm{C}-\mathrm{HA}_{1,90,30}$ & 5,41 & 2,16 \\
\hline P-HA $1,100,15$ & 48,22 & 19,29 & $\mathrm{C}-\mathrm{HA}_{1,100,15}$ & 7,04 & 2,82 \\
\hline $\mathrm{P}-\mathrm{HA}_{1,100,30}$ & 58,12 & 23,25 & $\mathrm{C}-\mathrm{HA}_{1,100,30}$ & 6,02 & 2,41 \\
\hline P-HA $1,120,15$ & $58,90 / 75,70$ & $23,56 / 30,28$ & $\mathrm{C}-\mathrm{HA}_{1,120,15}$ & $7,95 / 11,02$ & $3,18 / 4,41$ \\
\hline $\mathrm{P}-\mathrm{HA}_{1,120,30}$ & $70,20 / 80,04$ & $28,08 / 32,02$ & $\mathrm{C}-\mathrm{HA}_{1,120,30}$ & $7,21 / 12,02$ & $2,88 / 4,81$ \\
\hline P-HA $2,90,15$ & 43,86 & 17,54 & $\mathrm{C}-\mathrm{HA}_{2,90,15}$ & 5,25 & 2,10 \\
\hline $\mathrm{P}-\mathrm{HA}_{2,90,30}$ & 67,90 & 27,16 & $\mathrm{C}-\mathrm{HA}_{2,90,30}$ & 5,47 & 2,19 \\
\hline P-HA $2,100,15$ & 44,08 & 17,63 & $\mathrm{C}-\mathrm{HA}_{2,100,15}$ & 7,14 & 2,86 \\
\hline $\mathrm{P}-\mathrm{HA} \mathrm{A}_{2,100,30}$ & 51,62 & 20,65 & $\mathrm{C}-\mathrm{HA}_{2,100,30}$ & 7,47 & 2,99 \\
\hline $\mathrm{P}-\mathrm{HA}_{2,120,15}$ & $68,20 / 85,24$ & $27,28 / 34,10$ & $\mathrm{C}-\mathrm{HA}_{2,120,15}$ & $8,13 / 13,48$ & $3,25 / 5,39$ \\
\hline $\mathrm{P}-\mathrm{HA}_{2,120,30}$ & $62,28 / 60,34$ & $24,91 / 24,14$ & $\mathrm{C}-\mathrm{HA}_{2,120,30}$ & $7,89 / 11,74$ & $3,16 / 4,70$ \\
\hline
\end{tabular}

* 0 símbolo $\mathrm{P}$ na identificação dos ensaios corresponde ao uso da polpa madura $(\mathrm{P})$ nos ensaios de hidrólise ácida $(\mathrm{HA}) ; 0$ símbolo $\mathrm{C}$, às cascas. Os números sequenciais em subscrito na identificação de cada um dos ensaios representam a concentração de ácido sulfúrico $(0,1$ ou $2 \% \mathrm{~m} / \mathrm{m})$, temperatura $\left(90,100\right.$ ou $\left.120^{\circ} \mathrm{C}\right)$ e tempo de reação (15 ou $\left.30 \mathrm{~min}\right)$. ** Resultados separados por barras representam a repetição dos ensaios, realizada com novo lote de resíduo

\section{A.}

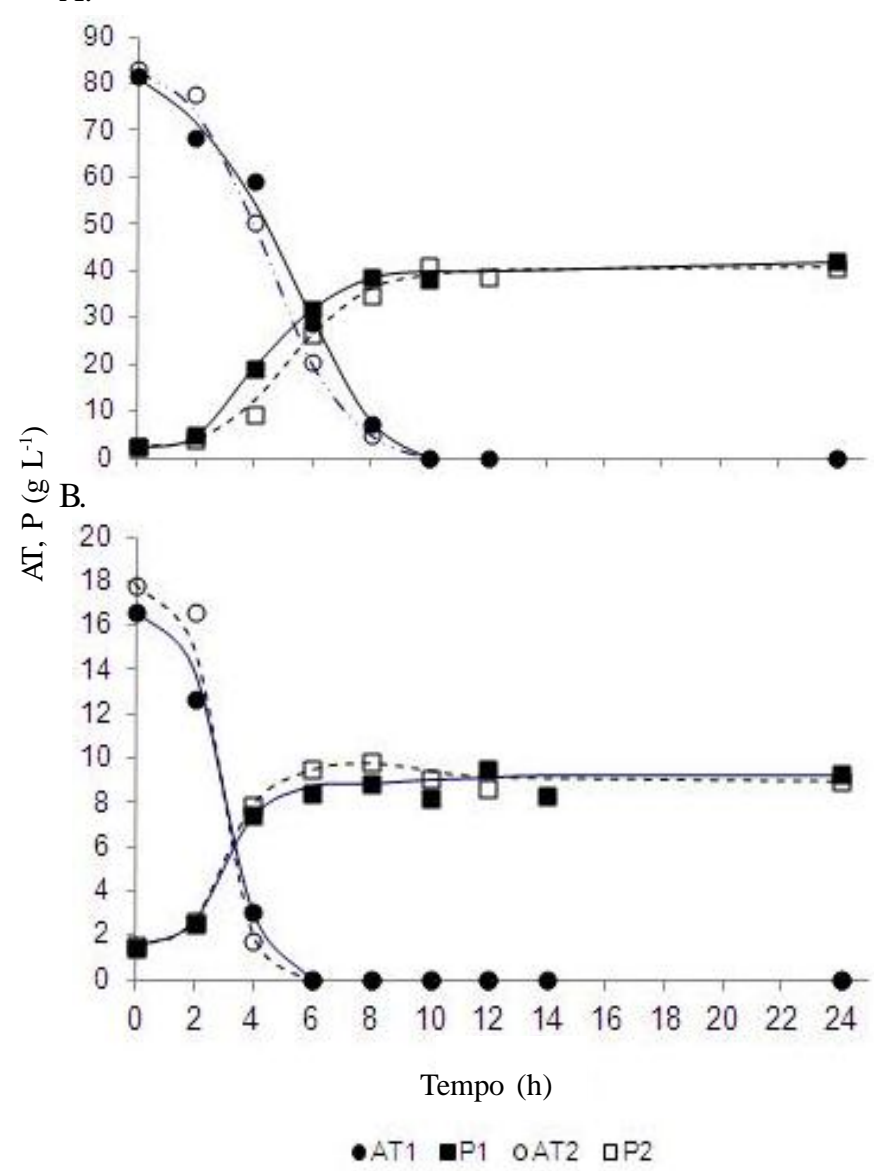

Obs.: AT1 e P1 correspondem aos valores médios observados no ensaio de fermentação e AT2 e P2, da sua replicata

Figura 1. Cinética do consumo de açúcares totais (AT) e produção de etanol $(\mathrm{P})$ para os ensaios de fermentação utilizando-se, como substrato, $500 \mathrm{~g} \mathrm{MU} \mathrm{L} \mathrm{L}^{-1}$ polpa madura de banana (A) e glicose (B) empregada em FGY $\left(20 \mathrm{gL}^{-1}\right)$, os autores utilizaram, como inóculo, $10 \mathrm{~g} \mathrm{MS} \mathrm{L}^{-1}$ de leveduras desidratadas provenientes de fermento comercial. Neste trabalho foram empregados, como inóculo, $20 \%$ $\mathrm{v} \mathrm{v}^{-1}$ de suspensão do mesmo micro-organismo, porém previamente cultivado durante $18 \mathrm{~h}$ no mesmo tipo de meio do caldo de fermentação. Apesar do tipo de inóculo utilizado neste trabalho conduzir a menor valor de $\mathrm{X}_{0}$ no caldo de fermentação $\left(\mathrm{X}_{0}=1,6 \mathrm{~g} \mathrm{MS} \mathrm{L}^{-1}\right)$, os microorganismos empregados já estavam adaptados ao meio de cultivo e se encontravam em condições biológicas de máximo crescimento celular aumentando, desta forma, a velocidade de consumo do substrato.

No ensaio $\mathrm{FPol}_{500}$ (Figura 1A), o tempo final de fermentação $\left(\mathrm{t}_{\mathrm{f}}\right)$ foi de $10 \mathrm{~h}$, com produção de etanol (P) de $39,7 \pm 2,1 \mathrm{~g} \mathrm{~L}^{-1} \mathrm{a}$ partir da concentração inicial de açúcares (AT) de $82,3 \pm 0,9$ $\mathrm{g} \mathrm{L}^{-1}$; para os demais ensaios com polpa esses valores foram da ordem de $\mathrm{t}_{\mathrm{f}}=6 \mathrm{~h}, \mathrm{P}=23,5 \pm 5,2 \mathrm{e} \mathrm{AT}=44,1 \pm 1,8 \operatorname{para~FPol}_{250} \mathrm{e}$ $\mathrm{t}_{\mathrm{f}}=9 \mathrm{~h}, \mathrm{P}=25,3 \pm 1,1$ e AT $=56,8 \pm 5,6 \mathrm{para} \mathrm{FPol}_{375^{\circ}}$.

Com a casca, conseguiu-se obter no máximo $\mathrm{AT}=15,9 \pm 5,1$ e $\mathrm{P}=7,5 \pm 0,5 \mathrm{~g} \mathrm{~L}^{-1}$ ao se utilizar biomassa na concentração de $1210 \mathrm{~g} \mathrm{MU} \mathrm{L}^{-1}$ (ensaio $\mathrm{FCas}_{1210}$ ). O valor de AT foi da ordem de apenas duas vezes maior do observado no ensaio $\mathrm{FCas}_{250}$ (AT $=7,6 \pm 0,7 \mathrm{~g} \mathrm{~L}^{-1}$ ) quando se esperava uma diferença de aproximadamente quatro vezes, discrepância passível de ser explicada devido principalmente a dois fatores: dificuldades operacionais no processo de extração do açúcar, especificamente na filtração manual em tecido de algodão com perda de substrato (enquanto no caso de $\mathrm{FCas}_{250}$ o substrato foi utilizado integralmente na composição do caldo de fermentação, em FCas $_{1210}$ os sólidos insolúveis grosseiros, provenientes das cascas, se mantiveram retidos no filtro saturados de líquido contendo açúcar) e propriedades diferentes entre os lotes de cascas utilizados em cada um dos ensaios (falta de padrão para emprego da fruta no mesmo estado de maturação). 
Em geral, foi possível observar que o aumento da concentração de biomassa conduziu ao aumento proporcional na concentração inicial de AT, exceto para o caso das cascas, e proporcionou o aumento da velocidade de consumo dos açúcares totais pelos microorganismos presentes no meio porém esse tipo de comportamento não se refletiu no rendimento, na produtividade nem na eficiência dos processos avaliados.

A Tabela 4 apresenta esses parâmetros obtidos a partir das respectivas cinéticas de cada ensaio de fermentação realizado.

Pode-se verificar, na Tabela 4, que não houve diferenças significativas $(\mathrm{P}<0,05)$ entre os rendimentos em etanol $\left(\mathrm{Y}_{\mathrm{P} / \mathrm{AT}}\right)$, produtividade total $\left(\mathrm{Q}_{\mathrm{P}}\right)$ e eficiência do processo $\left({ }^{\mathrm{a}}\right)$, obtidos em FPol e em FCas; portanto, dentro das condições experimentais avaliadas esses parâmetros não foram afetados pela quantidade de resíduo empregada no caldo de fermentação. A escolha da concentração ideal de cada um desses resíduos dependerá de alguns fatores, tais como: fluidez da mistura e análise do seu efeito sobre o consumo de energia para sua homogeneização (no caso de sistema agitado), custo da matériaprima, rendimento do processo de extração do produto do caldo fermentado (filtração e destilação) e quantidade de matéria residual gerada ao longo de todo o processo de obtenção do produto final. Essas variáveis serão objeto de estudo na continuidade deste trabalho.

Empregando os resíduos polpa e casca de banana Musa sp. em estado de maturação normal para consumo humano, Hammond et al. (1996) obtiveram rendimento médio em bioetanol de 0,116 e $0,019 \mathrm{~L} \mathrm{~kg}^{-1} \mathrm{MU}$, respectivamente. Considerando densidade do etanol de $789 \mathrm{~g} \mathrm{~L}^{-1}$ a $20{ }^{\circ} \mathrm{C}$ (Green \& Perry, 2008), esses valores corresponderam a 91,5 e 15,0 $\mathrm{g} \mathrm{kg}^{-1} \mathrm{MU}$, os quais foram semelhantes aos respectivos valores médios calculados a partir da relação P/MU para todos os ensaios FPol (80,3 \pm 13,3 $\left.\mathrm{g} \mathrm{kg}^{-1} \mathrm{MU}\right)$ e para oensaioFCas ${ }_{250}\left(14,5 \pm 0,8 \mathrm{~g} \mathrm{~kg}^{-1} \mathrm{MU}\right)$. Entretanto, para o caso do ensaio $\mathrm{FCas}_{1210}$, o rendimento em bioetanol obtido pelos pesquisadores foi da ordem de duas vezes maior que o valor médio alcançado neste trabalho $\left(8,2 \pm 0,5 \mathrm{~g} \mathrm{~kg}^{-1} \mathrm{MU}\right)$ diferença que pode ser explicada pela perda de substrato ocorrida no preparo do caldo de $\mathrm{FCas}_{1210}$, conforme já referido.

Sharma et al. (2007) empregaram uma mistura de casca de banana Musa sp. e cascas de tangerina, ambas secas e moídas para granulometria máxima de 40 mesh e obtiveram rendimento máximo de YP/AT $=0,426 \mathrm{~g} \mathrm{~g}^{-1} \mathrm{AT}$, valor este próximo ao encontrado neste trabalho (Tabela 4).

Manikandan et al. (2008) hidrolisaram previamente, utilizando apenas as cascas de banana secas e moídas, o resíduo com ácido sulfúrico e alcançaram concentração máxima de bioetanol no caldo fermentado igual a $9,8 \mathrm{~g} \mathrm{~L}^{-1}$ para uma concentração de $100 \mathrm{~g} \mathrm{~L}^{-1}$ de resíduo, ou seja, 0,098 $\mathrm{g} \mathrm{g}^{-1} \mathrm{MS}$. Considerando teor de umidade de 7,8 $\mathrm{g} \mathrm{H}_{2} \mathrm{O} \mathrm{g}^{-1} \mathrm{MS}$, o rendimento em base úmida de resíduo foi de $\mathrm{Y}_{\mathrm{P} / \mathrm{MU}}=11,1 \mathrm{~g} \mathrm{~kg}^{-1} \mathrm{MU}$, ou seja, menor que o alcançado em FCas $_{250^{\circ}}$.

Analisando os resultados de rendimento e a produtividade em bioetanol da fermentação da polpa e das cascas de banana madura em comparação a outros resíduos, é possível verificar que ambos são promissores, principalmente para a polpa de banana, cujos valores se aproximam daqueles obtidos com o caldo da cana-de-açúcar. A Tabela 5 apresenta essas comparações.

Tabela 5. Valores de rendimento e produtividade em bioetanol obtidos em fermentações de diferentes tipos de substrato

\begin{tabular}{lccl}
\hline \multicolumn{1}{c}{ Fonte de carbono } & $\begin{array}{c}\mathbf{Y}_{\mathrm{P} / \text { AT }} \\
\left(\mathbf{g ~ g}^{-1}\right)\end{array}$ & $\begin{array}{c}\mathbf{Q}_{\mathbf{P}} \\
\left(\mathbf{g ~ L}^{-1} \mathbf{h}^{-1}\right)\end{array}$ & \multicolumn{1}{c}{ Referência } \\
Cana-de-açúcar & 0,43 & 3,40 & Ribeiro \& Horii (1999) \\
Farelo de trigo & 0,38 & 1,92 & Palmarola-Adrados et al. (2005) \\
Cavacos de madeira & 0,40 & 0,67 & Sassner et al. (2006) \\
Farinha de milho & 0,50 & 1,21 & $\begin{array}{l}\text { Mojović et al. (2006) } \\
\text { Polpa de banana }\end{array}$ \\
& 0,48 & 3,04 & $\begin{array}{l}\text { Este trabalho, valor médio } \\
\text { calculado a partir dos valores de }\end{array}$ \\
Casca de banana & 0,34 & 1,32 & $\begin{array}{l}\text { FPol da Tabela 4 } \\
\text { Este trabalho, ECas }\end{array}$, Tabela 4 \\
\hline
\end{tabular}

Em função do baixo valor de rendimento alcançado com a casca de banana em relação aos demais substratos apresentados na Tabela 5, faz-se necessário a continuidade do estudo para o aumento deste parâmetro. Como consequência espera-se, com isto, o aumento também de $\mathrm{Q}_{\mathrm{P}}$. Em relação à polpa de banana pode-se observar que o substrato conduziu a valores de rendimento próximos aos obtidos por outros pesquisadores e à produtividade superior aos demais substratos empregados, inclusive em relação à cana-de-açúcar. Ribeiro \& Horii (1999) empregaram a cana-de-açúcar para uma concentração de 130 $\mathrm{g} \mathrm{L}^{-1}$ de sacarose e obtiveram aproxima-damente $55 \mathrm{~g} \mathrm{~L}^{-1} \mathrm{de}$ bioetanol $\left(7 \% \mathrm{v} \mathrm{v}^{-1}\right)$ após $16 \mathrm{~h}$ de fermentação enquanto neste trabalho foi empregado, para o caso da polpa de banana, o valor médio de $61,1 \mathrm{~g} \mathrm{~L}^{-1}$ de açúcar e obtida uma concentração final média de $29,8 \mathrm{~g} \mathrm{~L}^{-1}$ de bioetanol, após $8 \mathrm{~h}$ de processo. Esta diferença na concentração de bioetanol no caldo fermentado precisa ser avaliada com atenção uma vez que poderá implicar

Tabela 4. Valores médios de rendimento em etanol $\left(\mathrm{Y}_{\mathrm{P} / \mathrm{AT}}\right)$, produtividade total $\left(\mathrm{Q}_{\mathrm{P}}\right)$ e eficiência ( $\left.{ }^{\mathrm{a}}\right)$, obtidos na fermentação alcoólica da polpa de banana nanica madura (Pol) e da sua casca (Cas), ambas in natura, em biorreator de bancada (Ensaios F)

\begin{tabular}{|c|c|c|c|c|c|c|}
\hline \multirow{2}{*}{ Parâmetro } & \multicolumn{6}{|c|}{ Ensaios* } \\
\hline & $\mathrm{FG}^{(\mathrm{a}) * *}$ & $\mathrm{FPol}_{250}^{(\mathrm{b}) * *}$ & $\mathrm{FPol}_{375}{ }^{(\mathrm{c}) \star *}$ & $\mathrm{FPOI}_{500}{ }^{(\mathrm{d}) \star \star}$ & $\mathrm{FCas}_{250}^{(\mathrm{e}) * *}$ & FCas $_{1210}{ }^{(\mathrm{f}) * *}$ \\
\hline$Y_{P / A T}\left(g g^{-1}\right)$ & $\begin{array}{c}0,43 \pm 0,02 \\
b, c, d, e, f\end{array}$ & $\begin{array}{c}0,54 \pm 0,07 \\
\text { a,c,d,e,f }\end{array}$ & $\begin{array}{c}0,43 \pm 0,03 \\
\text { a,b,d,e,f }\end{array}$ & $\begin{array}{c}0,47 \pm 0,03 \\
\text { a,b,c,e,f }\end{array}$ & $\begin{array}{c}0,35 \pm 0,04 \\
a, c, f\end{array}$ & $\begin{array}{c}0,34 \pm 0,11 \\
a, b, c, d, e\end{array}$ \\
\hline$Q_{p}\left(g L^{-1} h^{-1}\right)$ & $\begin{array}{c}1,24 \pm 0,13 \\
b, e\end{array}$ & $\begin{array}{c}2,62 \pm 0,58 \\
\text { a,c,d,e }\end{array}$ & $\begin{array}{c}2,75 \pm 0,37 \\
b, d\end{array}$ & $\begin{array}{c}3,75 \pm 0,21 \\
b, c\end{array}$ & $\begin{array}{c}1,15 \pm 0,06 \\
a, b, f\end{array}$ & $\begin{array}{c}1,32 \pm 0,03 \\
a, b, e\end{array}$ \\
\hline$\epsilon(\%)$ & $\begin{array}{c}83,15 \pm 4,15 \\
\text { b,c,d,e,f }\end{array}$ & $\begin{array}{c}105,65 \pm 13,84 \\
\text { a,c,d,e,f }\end{array}$ & $\begin{array}{c}85,11 \pm 6,91 \\
\text { a,b,d,e,f }\end{array}$ & $\begin{array}{c}92,94 \pm 6,92 \\
a, b, c, e, f\end{array}$ & $\begin{array}{c}68,48 \pm 8,30 \\
\text { a,c,f }\end{array}$ & $\begin{array}{c}67,50 \pm 20,75 \\
\text { a,b,c,d,e }\end{array}$ \\
\hline
\end{tabular}

* Os números em subscrito na denominação dos ensaios representam a concentração de biomassa em massa úmida utilizada em cada experimento. ** Letras iguais às letras de cada ensaio, demonstram médias sem diferença significativa pelo método de Tukey, com nível de significância de 5\% (ANOVA) 
em diferenças significativas nos custos de purificação do produto. Os estudos do processo de destilação do bioetanol da polpa de banana estão em fase inicial de desenvolvimento.

\section{CONClusÕES}

1. O uso da hidrólise ácida da polpa ou das cascas não é recomendado para produção de bioetanol.

2. O aumento da concentração inicial da polpa de banana in natura de 250 para $500 \mathrm{~g} \mathrm{~L}^{-1}$ não proporcionou aumento no rendimento nem na produtividade em bioetanol; no entanto, o uso de $500 \mathrm{~g} \mathrm{~L}^{-1}$ proporcionou aumento de $50 \%$ na concentração do produto e no caldo fermentado.

3. A polpa de banana mostrou ter alto potencial para uso na produção de bioetanol.

\section{Agradecimentos}

Os autores agradecem à Fundação de Amparo à Pesquisa de Santa Catarina - FAPESC e ao Fundo de Apoio à Pesquisa da Universidade da Região de Joinville - FAP/UNIVILLE, pelo financiamento da pesquisa realizada.

\section{LiTERATURA CITADA}

ANEEL-Agência Nacional de Energia Elétrica. Atlas de energia elétrica do Brasil, 3.ed., Brasília: Brasília, 2008. 236p.

Arredondo, H. I. V.; Colorado, R. A.; Oliveira, S. Ethanol production from banana fruit and its lignocellulosic residues: Exergy and renewability analysis. International Journal of Thermodynamics, v.12, p.155-162, 2009.

Balat, M. Production of bioethanol from lignocellulosic materials via the biochemical pathway: A review. Energy Conversion and Management, v.52, p.858-875, 2011.

Campo, I. D.; Alegría, I.; Zazpe, M.; Echeverria, M.; Echeverria, I. Diluted acid hydrolysis pretreatment of agri-food wastes for bioethanol production, Industrial Crops and Products, v. 24, p. 214-221, 2006.

Cardona, C. A.; Sánchez, O. J. Fuel ethanol production: Process design trends and integration opportunities. Bioresource Technology, v.98, p.2415-2457, 2007.

Gaden Jr., E. C. Fermentation process kinetics. Journal of Biochemical and Microbial Technology Engineering, v.1, p.413-429, 1959.

Gonçalves, J. E.; Sartori, M. M. P.; Leão, A. L. Energia de briquetes produzidos com rejeitos sólidos urbanos e madeira de Eucalyptus grandis. Revista Brasileira de Engenharia Agrícola e Ambiental, v.13, p.657-661, 2009.

Green, D.; Perry, R. H. Perry's chemical engineers' handbook. 8ed. New York: McGraw-Hill, 2008. 2400p.

Hammond, J. B.; Egg, R.; Diggnins, D.; Coble, C. G. Alcohol from bananas. Bioresource Technology, v.56, p.125-130, 1996.
Leite, V. D.; Sousa, J. T.; Prasad, S.; Lopes, W. S.; Athayde Júnior, G. B.; Dantas, A. M. M. Tratamento de resíduos sólidos de centrais de abastecimento e feiras livres em reator anaeróbio de batelada. Revista Brasileira de Engenharia Agrícola e Ambiental, v.7, p.318-322, 2003.

Manikandan, K.; Saravanan, V.; Viruthagiri, T. Kinetics studies on ethanol production from banana peel waste using mutant strain of Saccharomyces cerevisiae. Indian Journal of Biotechnology, v.7, p.83-88, 2008.

Mohapatra, D.; Mishra, S.; Sutar, N. Banana and its by-product utilization: an overview. Journal of Scientific \& Industrial Research, v.69, p.323-329, 2010.

Mojoviæ, L.; Nikoliæ, S.; Rakin, M.; Vukasinoviæ, M. Production of bioethanol from corn meal hydrolyzates. Fuel, p.1750-1755, 2006.

Moreira, J. R. Obtenção de etanol a partir de material celulósico. In: Rosillo-Cale, F.; Bajay, S. V.; Rothman, H. (ed.). Uso da biomassa para produção de energia na indústria brasileira. Campinas: UNICAMP, 2005. 448p.

Palmarola-Adrados, B.; Chotìborská, P.; Galbe, M.; Zacchi, G. Ethanol production from non-starch carbohydrates of wheat bran. Bioresource Technology, v.96, p.843-850, 2005.

Quadros, D. G.; Oliver, A. P. M.; Valladares, R.; Souza, P. H. F.; Ferreira, E. J. Biodigestão anaeróbia de dejetos de caprinos e ovinos em reator contínuo de PVC flexível. Revista Brasileira de Engenharia Agrícola e Ambiental, v.14, p.326-332, 2010.

Ribeiro, C. A. F.; Horii, J. Potencialidades de linhagens de levedura Saccharomyces cerevisiae para a fermentação do caldo de cana. Scientia Agrícola, v.56, p.255-263, 1999.

Saito, I. M.; Cabello, C. Produção de etanol a partir de hidrolisado obtido por tratamento hidrotérmico de farelo de mandioca. Revista Energia na Agrícultura, v.21, p.34-44, 2006.

Sassner, P.; Galbe, M.; Zacchi, G. Bioethanol production based on simultaneous saccharification and fermentation of steampretreated salix at high dry-matter content. Enzyme and Microbial Technology, v.39, p.756-762, 2006.

Sassner, P.; Martensson, C. G.; Galbe, M.; Zacchi, G. Steam pretreatment of $\mathrm{H}_{2} \mathrm{SO}_{4}$ - impregnated Salix for the production of bioethanol. Bioresource Technology, v.99, p.137-145, 2007.

Sharma, N.; Kalra, K. L.; Oberoi, H. S.; Bansal, S. Optimization of fermentation parameters for production of ethanol from kinnow waste and banana peels by simultaneous saccharification and fermentation. Indian Journal of Microbiology, v.7, p.310-316, 2007.

Silva, D. J.; Queiroz, A. C. Análise de alimentos: Métodos químicos e biológicos. 3.ed., UFV: Viçosa, 2002. 235p.

Soest, P. J. van. Development of a comprehensive system of feed analysis and its applications to forage. Journal of Animal Science, v.26, p.119-128, 1967.

Solomon, B. D.; Barnes, J. R.; Halvorsen, K. E. Grain and cellulosic ethanol: History, economics, and energy policy. Biomass and Bionergy, v.31, p.416-425, 2007.

Souza, O.; Federizzi, M.; Coelho, B.; Wagner, T. M.; Wisbeck, E. Biodegradação de resíduos lignocelulósicos gerados na bananicultura e sua valorização para a produção de biogás. Revista Brasileira de Engenharia Agrícola e Ambiental, v.14, p.438-443, 2010.

Yu, Y. H.; Kim, S. D.; Lee, J. M.; Lee, K. H. Kinetic studies of dehydration, pyrolysis and combustion of paper sludge. Energy, v.27, p.457-469, 2002. 